\title{
DETERMINACIÓN DE LA ACTIVIDAD ANTIOXIDANTE DE EXTRACTOS DE HOJAS DE Buddleja inkana, Oreocallis grandiflora y Chuquiraga spinosa
}

\author{
Karen Tufinio Miranda a, Harold Ames Canchaya ${ }^{a}$, Abel Vergara Sotomayora, \\ Alejandro Fukusaki Yoshizawa ${ }^{\mathrm{b}}$, Karin Paucar Cuba ${ }^{* a}$
}

\begin{abstract}
RESUMEN
A fin de poder usar extractos de plantas nativas en aplicaciones medicinales, cosméticas, anticorrosivas, entre otras; el objetivo del presente trabajo fue evaluar la actividad antioxidante, así como el contenido de flavonoides y compuestos fenólicos totales de los extractos etanólicos e hidroalcohólicos de las hojas de Buddleja inkana (quisuar), Oreocallis grandiflora (cucharilla) y Chuquiraga spinosa (huamanpinta). La medición de la actividad antioxidante: FRAP, DPPH y ABTS ${ }^{+}$así como el contenido de flavonoides y compuestos fenólicos totales, se realizó por el método de lector en microplaca. Los resultados obtenidos al evaluar la actividad antioxidante de los extractos en etanol absoluto y en soluciones hidroalcohólicas al 75 y $50 \%(\mathrm{v} / \mathrm{v})$ en etanol mostraron que los extractos de la Oreocallis grandiflora presentaron una mayor actividad antioxidante comparados con los extractos de la Buddleja inkana y Chuquiraga spinosa, y que los extractos hidroalcohólicos al 75 $\%(\mathrm{v} / \mathrm{v})$ de etanol fueron los extractos con la mayor actividad antioxidante, probablemente asociado al solvente y a que dichos extractos presentaron también mayores concentraciones de compuestos fenólicos totales.

Palabras clave: Buddleja inkana, Oreocallis grandiflora, Chuquiraga spinosa, antioxidantes, flavonoides, fenoles.

\section{DETERMINATION OF THE ANTIOXIDANT ACTIVITY OF Buddleja inkana, Oreocallis grandiflora and Chuquiraga spinose LEAF EXTRACTS}

\begin{abstract}
In order to use native plant extracts in medicinal, cosmetic, anticorrosive and other applications; the objective of these work was to evaluate the antioxidant activity, as well as the content of flavonoids and total phenolic compounds of the ethanolic and hydroalcoholic

\footnotetext{
${ }^{a}$ Laboratorio de Corrosión. Facultad de Ingeniería Química y Textil. Universidad Nacional de Ingeniería-UNI. Av. Túpac Amaru N²10. Lima 25. kpaucar@uni.edu.pe.

${ }^{\text {b }}$ Laboratorio de Química y Bioquímica de Productos Naturales. Universidad Científica del Sur-UCSUR.
} 
extracts of the leaves of Buddleja inkana (quisuar), Oreocallis grandiflora (cucharilla) and Chuquiraga spinose (huamanpinta). The measurement of antioxidant activity: FRAP, DPPH and ABTS $\cdot^{+}$, as well as the content of flavonoids and total phenolic compounds, was carried out by the microplate reader method. The results when evaluating the antioxidant activity of ethanolic and hydroalcoholic solutions at 75 and $50 \%(\mathrm{v} / \mathrm{v})$ in ethanol extracts showed that the extracts of Oreocallis grandiflora showed a higher antioxidant activity than Buddleja inkana and Chuquiraga spinosa, and that the hydroalcoholic extracts at $75 \%(\mathrm{v} / \mathrm{v})$ ethanol were the extracts with high antioxidant activity, probably associated with the solvent, since these extract also presented the highest concentrations of total phenolic compounds.

Key words: Buddleja inkana, Oreocallis grandiflora, Chuquiraga spinosa, antioxidants, flavonoids, phenols.

\section{INTRODUCCIÓN}

En el Perú, la identificación e investigación en los usos de plantas nativas es todavía incipiente en comparación a la extensa flora con la que contamos debido a nuestra diversidad geográfica y climática; lo cual motiva a profundizar su estudio. Con la finalidad de poder valorar sus propiedades e importancia, la mayor parte de investigaciones se basan en la búsqueda de plantas con propiedades alimenticias, medicinales, anticorrosivas, entre otras ${ }^{1,2}$. La actividad antioxidante de sus extractos y/o sus fracciones, es muy estudiada en farmacología y se ha visto su relación con el contenido de fenoles y flavonoides presentes en algunas plantas ${ }^{3}$.

Por otro lado, los ensayos para la determinación de la actividad antioxidante pueden clasificarse según la naturaleza del mecanismo sobre el cual los antioxidantes neutralizan los radicales libres; de esta forma, existen ensayos basados en la transferencia de átomos de hidrógeno y ensayos basados en la transferencia de electrones. A este segundo tipo pertenecen los análisis TEAC (Capacidad antioxidante de equivalentes TROLOX), FRAP (parámetro antioxidante de la reducción del ion férrico), DPPH (2,2-difenil-2-picrihidrazil), capacidad de reducción del cobre II y ensayo de fenoles totales por reactivos de Folin Ciocalteu.

Los métodos espectroscópicos basados en la transferencia de electrones, miden la capacidad de un antioxidante de reducir un agente oxidante, ocurriendo generalmente un cambio de color (el cambio de color es directamente proporcional a la concentración de los antioxidantes de la muestra) sobre el compuesto reducido, los casos más representativos son la pérdida de color de los ensayos realizados con el $\mathrm{DPPH}^{4}$ y el radical catión $\mathrm{ABTS}^{+{ }^{+}}$. El objetivo del presente estudio fue determinar el contenido de flavonoides, compuestos fenólicos totales y la actividad antioxidante de extractos de diferente polaridad de tres plantas nativas: Buddleja inkana, Oreocallis grandiflora y Chuquiraga spinosa, con fines medicinales, cosméticos, anticorrosivos, etc., se aplicó el método de microplaca para la determinación de flavonoides, fenoles totales y los ensayos de inhibición de radicales FRAP, DPPH y ABTS ${ }^{++}$. 


\section{PARTE EXPERIMENTAL}

\section{Obtención de las muestras}

La muestra de Buddleja inkana Ruiz \& Pav., de la familia Loganiaceae (quisuar); fue recolectada en la provincia de Canta (Lima) e identificada por el biólogo Severo Baldeón Malpartida (constancia No. 072-USM-2018). La muestra de Oreocallis grandiflora (Lam) R. Br., de la familia Proteaceae, fue recolectada en marzo del 2018 en la carretera Tiambra, hacia arriba de la quebrada, km 14 desde Huasahuasi, provincia de Tarma (Junín). Es un arbusto de 1-2 m de alto, con flores de color rosado fucsia y su hábitat es matorral; fue identificada por la bióloga Asunción Cano Echevarría (constancia No. 071-USM-2018). La muestra de Chuquiraga spinosa Less "huamanpita", de la familia Astereaceae, fue recolectada en febrero del 2018 en el distrito de Huaros, provincia de Canta (Lima). Es un arbusto espinoso con 0,51,5 $\mathrm{m}$ de alto, flores anaranjadas, crece en matorral en zona rocosa dominada por arbustos de Baccharis sp. y Lupinus sp.; fue identificada en el Museo de Historia Natural de la UNMSM por el biólogo Severo Baldeón Malpartida (constancia No. 070-USM-2018).

\section{Obtención de los extractos}

Para la preparación de los extractos se seleccionó solo las hojas de la muestra bruta de cada planta: Buddleja incaka, Oreocallis grandiflora y Chuquiraga spinosa. Para la extracción por maceración las hojas fueron limpiadas, secadas bajo sombra durante 21 días, molidas con un molino de mano y tamizadas en tamiz $\mathrm{N}^{\circ}$ 40. Para la extracción se utilizó $50 \mathrm{~g}$ de la muestra molida en $100 \mathrm{~mL}$ de solvente, es decir, etanol absoluto y soluciones hidroalcóholicas al 75 y $50 \%(\mathrm{v} / \mathrm{v})$ en etanol. La maceración se realizó en caliente a $40{ }^{\circ} \mathrm{C}$ durante 4 días, en este periodo de tiempo la muestra se sonicó y filtró 5 veces, las primeras por gravedad usando papel filtro rápido y la última por filtración al vacío; al cuarto día se obtuvo el extracto líquido que fue secado en una estufa a $40{ }^{\circ} \mathrm{C}$ hasta obtener el extracto sólido. Los extractos, así obtenidos, fueron utilizados para realizar las pruebas de actividad antioxidante: FRAP, DPPH y ABTS ${ }^{+}$, así como la determinación de flavonoides y compuestos fenólicos totales.

\section{Tamizaje fitoquímico}

El tamizaje fitoquímico, conocido también como marcha fitoquímica, es un método cualitativo que permite identificar, de manera preliminar, los diferentes componentes o metabolitos secundarios presentes en una planta. El tamizaje fitoquímico de las tres plantas se realizó usando $10 \mathrm{~g}$ de las hojas secas, molidas y tamizadas de cada planta y siguiendo los procedimientos descritos por Lock7 para la identificación de metabolitos en cada planta.

\section{Determinación de antioxidantes}

Se empleó como solvente al metanol absoluto, se preparó $3 \mathrm{~mL}$ de la solución de cada extracto de las plantas: Buddleja inkana, Oreocallis grandiflora y Chuquiraga spinosa a una concentración de $5 \mathrm{mg} / \mathrm{mL}$ en metanol. Se pesó $150 \mathrm{mg}$ de cada muestra, se llevó al ultrasonido por $5 \mathrm{~min}$, después se introdujo en un vórtex. Las plantas con su respectiva concentración en etanol e hidroalcohólicas se identificaron; en etanol absoluto: Buddleja inkana (B ABS.), Oreocallis grandiflora (O ABS.), y Chuquiraga Spinosa (C ABS.), al 75 \% (v/v) en etanol: Buddleja inkana (B 75), Oreocallis grandiflora (O 75) y Chuquiraga spinosa 
(C 75) y al $50 \%$ (v/v) en etanol: Buddleja inkana (B 50), Oreocallis grandiflora (O 50) y Chuquiraga spinosa (C 50). Las mezclas, ya disueltas, se pasaron por un filtro de $22 \mu \mathrm{m}$ y se realizó las pruebas de FRAP, DPPH, $\mathrm{ABTS}^{\cdot}{ }^{+}$, flavonoides y compuestos fenólicos totales ${ }^{8}$.

Poder Antioxidante de Reducción Férrica (FRAP), en lector de micro placa (modificado para etanol): Este ensayo se basa en la capacidad de reducir el ion $\mathrm{Fe}^{+3} \mathrm{a} \mathrm{Fe}^{+2}$ en presencia del TPTZ (6-hidroxi-2,5,7,8-tetrametilcroman-2-ácido carboxílico) formando un complejo azul intenso de Fe-TPTZ con una absorbancia máxima de $593 \mathrm{~nm}$. La absorbancia es proporcional al contenido de antioxidantes.

Para el ensayo se mezcló $290 \mu \mathrm{L}$ de solución de trabajo (preparada a partir de $1 \mathrm{~mL}$ de solución tampón $300 \mathrm{mM}\left(3,1 \mathrm{~g}\right.$ de $\mathrm{CH}_{3} \mathrm{COONa} \cdot 3 \mathrm{H}_{2} \mathrm{O}$ y $16 \mathrm{~mL}$ de ácido acético glacial), 1 $\mathrm{mL}$ de $20 \mathrm{mM} \mathrm{FeCl} \cdot 6 \mathrm{H}_{2} 0$ en solución acuosa en $0,02 \mathrm{M} \mathrm{HCl}, 1 \mathrm{~mL}$ de $10 \mathrm{mM}$ TPTZ disuelto en etanol y $11 \mathrm{~mL}$ de etanol) y $10 \mu \mathrm{L}$ de muestra en un pocillo de microplaca, se esperó 15 min y se midió la absorbancia a $593 \mathrm{~nm}$. Las mediciones se realizaron por triplicado. La curva de calibración se obtuvo usando una solución de Trolox, ver figura 1. Los resultados se expresaron en mg de equivalentes de Trolox/g de planta.

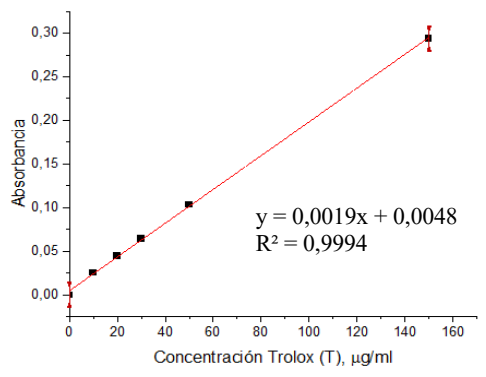

Figura 1. Curva de calibración de Trolox.

Inhibición frente al radical libre 2,2-difenil-1-picrilhidrazilo (DPPH) en lector de micro placa: Para el ensayo se mezcló $150 \mu \mathrm{L}$ de disolución de $400 \mu \mathrm{M}$ DPPH (2,2-difenil-1picrilhidrazilo) en metanol y $50 \mu \mathrm{L}$ de la muestra en un pocillo de microplaca, se esperó 30 minutos y se midió la absorbancia a $515 \mathrm{~nm}$. Las muestras se midieron por triplicado. Se empleó como estándar de control el ácido gálico.

Decoloración del radical $\mathrm{ABTS}^{+}{ }^{+}$ácido 2,2'-azino-bis (3-etilbenzotiazolina-6-ácido sulfónico)) en lector de micro placa: Para el ensayo, se prepararon $5 \mathrm{~mL}$ de solución acuosa $7 \mathrm{mM}$ de ABTS 2,2'-azino-bis (3-ethylbenzothiazoline-6-sulphonic acid) y $5 \mathrm{~mL}$ de solución acuosa $2,45 \mathrm{mM}$ de persulfato de potasio, las cuales luego se mezclaron en proporciones (1:1) y se dejó incubar por 16 h en oscuridad, generando una disolución color verde cuya absorbancia se midió a $734 \mathrm{~nm}$. Una alícuota de dicha solución se diluyó con una solución 
tampón fosfato salino hasta alcanzar un valor de absorbancia de 0,7 . Para el ensayo se mezcló $275 \mu \mathrm{L}$ de dicha solución y $25 \mu \mathrm{L}$ de muestra en un pocillo de microplaca y luego de 5 min de reacción se midió la absorbancia a $734 \mathrm{~nm}$. Se usó como estándar de control el ácido gálico.

La ecuación 1 permite calcular el porcentaje de actividad antioxidante (AA) de los extractos para los ensayos DPPH y ABTS $\cdot{ }^{+}$:

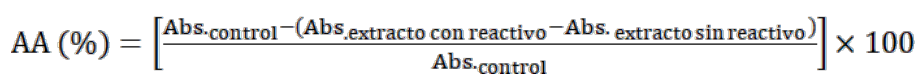

En base a las curvas de concentración vs. porcentaje de actividad antioxidante se obtuvieron los coeficientes de inhibición al $50 \%$ de actividad, $\mathrm{IC}_{50}$, de los extractos y del estándar control. La determinación del porcentaje de actividad antioxidante relativa (\% AAR) de los extractos de hojas para los ensayos DPPH y ABTS se realizó usando la ecuación 2:

$$
\operatorname{AAR}(\%)=\mathrm{IC}_{50(\mathrm{M})} / \mathrm{IC}_{50(\mathrm{R})} * 100
$$

Donde:

$\mathrm{IC}_{50(\mathrm{M})}$ : Coeficiente de inhibición de la muestra.

$\mathrm{IC}_{50(\mathrm{R})}$ : Coeficiente de inhibición del estándar de control.

Flavonoides en lector de micro placa: Los flavonoides son los más comunes antioxidantes, el estándar a comparar fue la quercetina, ver figura 2. Para el ensayo se mezcló $160 \mu \mathrm{L}$ de solución acuosa de $\mathrm{NaNO}_{2} 0,85 \%$ p/v y $30 \mu \mathrm{L}$ de muestra en un pocillo de la microplaca y se esperó 6 min. Se agregó $18 \mu \mathrm{L}$ de solución acuosa de $\mathrm{AlCl} 310 \%$ y se esperó 5 min. Posteriormente se agregó $120 \mu \mathrm{L}$ de mezcla básica (preparada usando $1 \mathrm{~mL}$ de etanol absoluto con 3,1 mL de $\mathrm{NaOH}$ 0,645 M). Se homogenizó la mezcla mediante pipeteo y se medió inmediatamente la absorbancia a $510 \mathrm{~nm}$. Las muestras se midieron por triplicado. Los resultados fueron expresados en mg quercetina equivalente (AG)/g de planta.

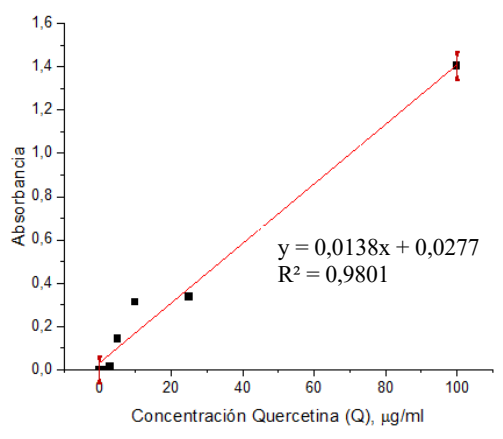

Figura 2. Curva de calibración de Quercetina. 
Fenoles totales en lector de micro placa: Para el ensayo se mezcló $165 \mu \mathrm{L}$ de solución de reactivo Folin-Cicalteu (preparada usando $1 \mathrm{~mL}$ del reactivo en $139 \mathrm{~mL}$ de agua) con 15 $\mu \mathrm{L}$ de muestra en un pocillo de microplaca, se esperó 5 min. Se agregó $120 \mu \mathrm{L}$ de solución acuosa de $\mathrm{Na}_{2} \mathrm{CO}_{3} 7 \%$ p/v y se midió a $750 \mathrm{~nm}$. Se realizó la curva de calibración con una solución alcohólica de ácido gálico (AG), ver figura 3. Los resultados fueron expresados en mg ácido gálico equivalente $(\mathrm{AG}) / \mathrm{g}$ de planta. Este procedimiento se efectuó por triplicado con cada uno de los extractos.

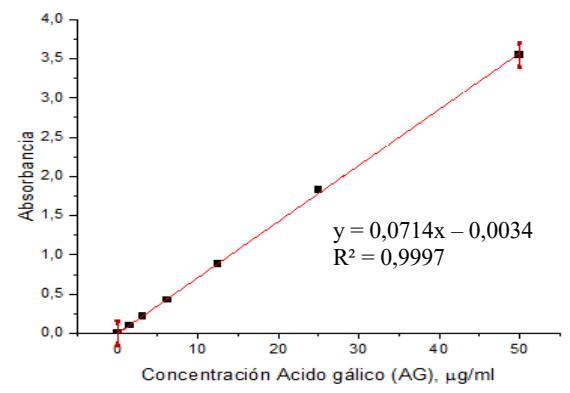

Figura 3. Curva de calibración de ácido gálico.

\section{RESULTADOS Y DISCUSIÓN}

\section{Obtención de los extractos}

La cantidad de extracto obtenido para cada planta en solución fue: 2, 8, 4,7 y 2,5 g para B ABS., B 75 y B 50, respectivamente; 1,7, 1,9 y 4,1 g, para O ABS., O 75 y O 50, respectivamente; 1,5, 2,5 y 1,5 g, para C ABS., C 75 y C 50, respectivamente. En general, la Buddleja inkana fue la planta que mostró la mayor extracción con los diferentes solventes y que los solventes hidroalcohólicos favorecieron la extracción de metabolitos, probablemente debido a la solubilidad de algunos metabolitos en presencia de agua.

\section{Tamizaje fitoquímico}

Los resultados obtenidos en la determinación de la composición química de las distintas fracciones de cada una de las plantas estudiadas se muestran en la tabla 1. El tamizaje fitoquímico mostró la presencia de fenoles, taninos, esteroides, triterpenoides, alcaloides, saponinas y leucoantocianinas, metabolitos que pueden ser usados en diversas aplicaciones ${ }^{1,2}$. 
Tabla 1. Tamizaje fitoquímico.

\begin{tabular}{|c|c|}
\hline Hojas de la planta & Metabolitos secundarios \\
\hline Buddleja inkana & $\begin{array}{l}\text { Componentes fenólicos, taninos, saponinas, triterpenoides, } \\
\text { alcaloides. }\end{array}$ \\
\hline Oreocallis grandiflora & $\begin{array}{l}\text { Componentes fenólicos, taninos, flavonoides, saponinas, } \\
\text { triterpenoides, estereoides, leucoantocianinas, alcaloides. }\end{array}$ \\
\hline Chuquiraga spinosa & $\begin{array}{l}\text { Componentes fenólicos, flavonoides, alcaloides, } \\
\text { leucoantocianinas. }\end{array}$ \\
\hline
\end{tabular}

\section{Actividad antioxidante}

\section{Ensayo de FRAP (Ferric reducing ability power)}

Los extractos etanólicos e hidroalcohólicos de la Buddleja inkana y Oreocallis grandiflora, mostraron una intensa coloración azul debido a la formación del complejo mientras que los extractos de Chuquiraga spinosa el cambio de coloración no fue observada a simple vista, lo cual guarda relación con el mayor rendimiento de los extractos, así como en los valores de actividad antioxidante reportados en la tabla 2. Los extractos con la mayor actividad antioxidante, en todos los casos, fueron los extractos hidroalcohólicos B 75, O 75 y C 75 con valores de $1997,78,1317,53$, y 56,98 $\mathrm{mg}$ trolox/g planta, respectivamente.

El mayor valor de la actividad antioxidante de los extractos hidroalcohólicos en comparación con los etanólicos podría atribuirse al solvente de extracción utilizado, es decir, que la mezcla de solventes (etanol/agua), en ciertas composiciones, favorecen la extracción de metabolitos secundarios con propiedades antioxidantes. Otros estudios realizados en extractos metanólicos de las hojas de la Buddleja saligna reportaron valores de actividad antioxidante FRAP de $490,98 \mu \mathrm{mol} \mathrm{Fe}$ (II)/g de muestra ${ }^{9}$.

Tabla 2. Actividad antioxidante FRAP de extractos etanólicos e hidroalcohólicos de Buddleja inkana, Oreocallis grandiflora y Chuquiraga spinosa.

\begin{tabular}{cc}
\hline planta/solvente & $\begin{array}{c}\text { Actividad antioxidante } \\
\text { (mg trolox/g muestra) }\end{array}$ \\
\hline B ABS. & $899,56 \pm 0,01$ \\
B 75 & $1997,78 \pm 0,01$ \\
B 50 & $941,78 \pm 0,01$ \\
O ABS. & $1106,97 \pm 0,03$ \\
O 75 & $1317,53 \pm 0,04$ \\
O 50 & $870,73 \pm 0,01$ \\
C ABS. & $10,04 \pm 0,01$ \\
C 75 & $56,98 \pm 0,01$ \\
C 50 & $25,20 \pm 0,01$ \\
\hline
\end{tabular}




\section{Ensayo DPPH}

En la tabla 3, se muestra los IC50 y el AAR (\%) de los extractos etanólicos e hidroalcohólicos estudiados, así como del estándar control, evidenciando que los extractos hidroalcohólicos $\mathrm{O}$ 75, B 75 y C 75 exhiben una mejor actividad antioxidante por este método comparado con los demás extractos estudiados.

En particular, la actividad antioxidante por DPPH de los extractos de Oreocallis grandiflora fue mayor que la exhibida por los extractos de Buddleja inkana. Comparando los valores de $\mathrm{IC}_{50}$ para los extractos estudiados, el valor de $25,26 \mu \mathrm{g} / \mathrm{mL}$ fue el más bajo alcanzado por el extracto $\mathrm{O}$ 75. Sin embargo, no tiene una buena capacidad antioxidante respecto al estándar patrón de ácido gálico.

Los extractos que mostraron la mayor actividad antioxidante relativa fueron los extractos $\mathrm{O}$ 75 y O ABS., con valores de 432,67 \% y 632,75\%, respectivamente; verificando que estos menores valores exhiben una mayor capacidad de captar los radicales libres.

Ensayos de DPPH a extractos etanólico de la Buddleja saligna ${ }^{10}$, metanólico de la Buddleja globosa ${ }^{11}$ e hidrofenil etil vainillato de la Buddleja salvifiola ${ }^{12}$ han reportado valores de $\mathrm{IC}_{50}$ de $8,16,8,40$ y $84,03 \mu \mathrm{g} / \mathrm{mL}$, respectivamente; todos estos valores difieren del solvente, especies y partes de plantas usadas pertenecen a la familia de la Buddleja inkana, la cual tiene aún estudios incipientes.

Tabla 3. Coeficiente de inhibición, IC50, y Actividad antioxidante relativa (AAR) mediante DPPH de los extractos etanólicos e hidroalcohólicos de Buddleja inkana, Oreocallis grandiflora y Chuquiraga spinosa.

\begin{tabular}{crc}
\hline planta/solvente & \multicolumn{1}{c}{$\begin{array}{c}\mathbf{I} \mathbf{C}_{\mathbf{5 0}} \\
(\boldsymbol{\mu} \mathbf{m L})\end{array}$} & $\begin{array}{c}\text { AAR } \\
(\mathbf{\%})\end{array}$ \\
\hline B ABS. & $72,84 \pm 0,01$ & 1247,58 \\
B 75 & $62,39 \pm 0,01$ & 1068,59 \\
B 50 & $67,02 \pm 0,03$ & 1147,89 \\
O ABS. & $36,94 \pm 0,01$ & 632,75 \\
O 75 & $25,26 \pm 0,01$ & 432,67 \\
O 50 & $122,14 \pm 0,02$ & 2091,83 \\
C ABS. & $3439,09 \pm 0,01$ & 58899,71 \\
C 75 & $773,28 \pm 0,01$ & 12558,59 \\
C 50 & $1361,61 \pm 0,01$ & 23319,72 \\
Acido Gálico & $5,83 \pm 0,04$ & --- \\
\hline
\end{tabular}

Otros estudios realizados a extractos metanólicos de la Oreocallis grandiflora ${ }^{13}$ reportaron, en ensayos DPPH, valores de IC50 de $15 \mu \mathrm{g} / \mathrm{mL}$ concentraciones menores a las obtenidas para los extractos estudiados. Por otro lado, estudios realizados a extractos etanólicos de Chiquiraga spinosa ${ }^{14}$ y metanólicos de Chuquiraga spinosa ${ }^{15}$, reportaron resultados con una mayor actividad antioxidante, con valores de actividad antioxidante, $\mathrm{IC}_{50}$, de 14,98 y 36,51 
$\mu \mathrm{g} / \mathrm{mL}$, respectivamente. El menor valor de $\mathrm{IC}_{50}$ probablemente se encuentre relacionado con las condiciones de extracción, lugar de origen, entre otros.

\section{Ensayo de ABTS $^{\cdot+}$}

En la tabla 4 se muestra los $\mathrm{IC}_{50}$ y AAR (\%) de los extractos estudiados, así como del estándar control, evidenciando que los extractos O 75, B 75 y C 75, al igual que en el ensayo DPPH, exhiben una mejor actividad antioxidante comparado con los demás extractos estudiados. De forma similar a lo evidenciado en los ensayos anteriores, la actividad antioxidante por ABTS de los extractos de Oreocallis grandiflora fue mayor que la exhibida por los extractos de Buddleja inkana. Comparando los valores de $\mathrm{IC}_{50}$ para los extractos estudiados, el valor de 4,69 $\mu \mathrm{g} / \mathrm{mL}$ fue el más bajo alcanzado para el extracto O 75. Sin embargo, no tiene una buena capacidad antioxidante respecto al estándar patrón de ácido gálico. Los extractos que mostraron la mayor actividad antioxidante relativa fueron los extractos $\mathrm{O} 75$ y O ABS., con valores de 9,60 y 10,84 \%, respectivamente; verificando que a menor valor mayor capacidad antioxidante.

Tabla 4. Coeficiente de inhibición, $\mathrm{IC}_{50}, \mathrm{y}$ Actividad antioxidante relativa (AAR) mediante ABTS de los extractos etanólicos e hidroalcohólicos de Buddleja inkana, Oreocallis grandiflora y Chuquiraga spinosa.

\begin{tabular}{ccc}
\hline planta/solvente & $\begin{array}{c}\mathbf{I C}_{\mathbf{5}} \\
(\boldsymbol{\mu g} / \mathbf{m L})\end{array}$ & $\begin{array}{c}\text { AAR } \\
(\mathbf{\%})\end{array}$ \\
\hline B ABS. & $8,33 \pm 0.01$ & 17,06 \\
B 75 & $6,54 \pm 0.01$ & 13,38 \\
B 50 & $7,25 \pm 0.01$ & 14,82 \\
O ABS. & $5,30 \pm 0.01$ & 10,84 \\
O 75 & $4,69 \pm 0.01$ & 9,60 \\
O 50 & $11,31 \pm 0.01$ & 23,14 \\
C ABS. & $90,07 \pm 0.01$ & 184,23 \\
C 75 & $37,13 \pm 0.01$ & 75,96 \\
C 50 & $52,08 \pm 0.01$ & 106,53 \\
Ácido Gálico & $0,48 \pm 0.01$ & --- \\
\hline
\end{tabular}

Los valores de AAR (\%) reportados para extractos metanólicos y etanólicos de la Calamintha grandiflora $^{16}$, especie perteneciente a la misma división y especie de la Oreocallis grandiflora, fueron de 18,67 y $23,56 \%$, respectivamente; indicando una mayor actividad antioxidante que los extractos de hojas de la Oreocallis grandiflora.

En la figura 4, se muestra que los valores de la concentración de los extractos para $\mathrm{IC}_{50}$, calculados por el ensayo de DPPH son mayores que los calculados para el ensayo de ABTS ${ }^{\circ+}$. El ensayo ABTS muestra una mayor sensibilidad a la actividad antioxidante. Los extractos $\mathrm{O} 75$ y O ABS. mostraron los valores de IC50 más bajos para $\mathrm{ABTS}^{\cdot+}$ y una mayor actividad antioxidante por ambos métodos. 


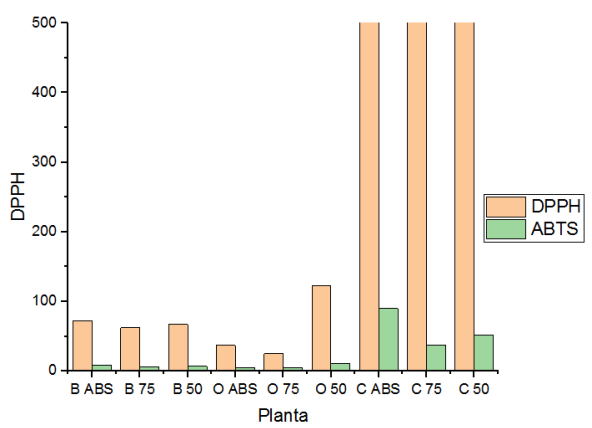

Figura 4. Actividad antioxidante por ensayos de DPPH y $\mathrm{ABTS}^{\cdot+}$.

\section{Contenido de flavonoides y fenoles totales}

En la tabla 5, se muestra los resultados obtenidos en la medición del contenido de flavonoides. Los extractos etanólicos e hidroalcohólicos de la Buddleja inkana mostraron de 2 a 3 veces mayor el contenido de flavonoides que los extractos de Oreocallis grandiflora y Chuquiraga spinosa.

Tabla 5. Contenido de flavonoides de extractos etanólicos e hidroalcohólicos de Buddleja inkana, Oreocallis grandiflora y Chuquiraga spinosa.

\begin{tabular}{cccc}
\hline planta/solvente & Absorbancia & Desv. Estándar & $\begin{array}{c}\text { Flavonoides } \\
\text { mg Quercetina/g planta }\end{array}$ \\
\hline B ABS. & 1,58 & 0,09 & $210,68 \pm 0,09$ \\
B 75 & 1,66 & 0,07 & $369,24 \pm 0,07$ \\
B 50 & 1,02 & 0,10 & $118,52 \pm 0,10$ \\
O ABS. & 0,53 & 0,01 & $39,97 \pm 0,01$ \\
O 75 & 0,74 & 0,01 & $65,14 \pm 0,01$ \\
O 50 & 0,18 & 0,09 & $31,44 \pm 0,09$ \\
C ABS. & 0,50 & 0,04 & $34,14 \pm 0,04$ \\
C 75 & 0,96 & 0,28 & $111,11 \pm 0,28$ \\
C 50 & 0,10 & 0,16 & $5,51 \pm 0,16$ \\
\hline
\end{tabular}

En la tabla 6, se muestra los resultados obtenidos en la medición del contenido de fenoles totales. Los extractos alcohólicos e hidroalcohólicos al $75 \%(\mathrm{v} / \mathrm{v})$ de etanol de las tres plantas en estudio mostraron los mayores contenidos de flavonoides y polifenoles totales, resultado que mantiene correlación con la elevada actividad antioxidante reportada en los ensayos FRA, DPPH y ABTS ${ }^{+}$. 
Tabla 6. Contenido de fenoles totales de extractos etanólicos e hidroalcohólicos de Buddleja inkana, Oreocallis grandiflora y Chuquiraga spinosa.

\begin{tabular}{cccc}
\hline planta/solvente & Absorbancia & Desv. Estándar & $\begin{array}{c}\text { Fenoles totales } \\
\text { mg Ácido Gálico/g planta }\end{array}$ \\
\hline B ABS. & 1,75 & 0,05 & $91,77 \pm 0,05$ \\
B 75 & 1,97 & 0,10 & $172,58 \pm 0,10$ \\
B 50 & 1,79 & 0,10 & $82,29 \pm 0,10$ \\
O ABS. & 2,94 & 0,10 & $89,88 \pm 0,10$ \\
O 75 & 2,65 & 0,02 & $93,09 \pm 0,02$ \\
O 50 & 1,02 & 0,06 & $78,46 \pm 0,06$ \\
C ABS. & 0,34 & 0,05 & $9,63 \pm 0,05$ \\
C 75 & 0,54 & 0,01 & $24,89 \pm 0,01$ \\
C 50 & 0,29 & 0,01 & $8,50 \pm 0,01$ \\
\hline
\end{tabular}

El contenido de fenoles para extractos metanólicos de la Oreocallis grandiflora ${ }^{13}$ reportaron valores de 17,4 mg Ácido Gálico/100 mg de extracto, este resultado es tres veces menor al obtenido para el extracto etanólico en estudio. Otros estudios de contenido de flavonoides realizados solo a extractos etanólicos al $96 \%$ de hojas de Chuquirga spinosa $a^{17}$ obtuvieron como resultado $80 \mathrm{mg}$ quercetina/g y en el extracto de estudio se obtuvo entre 5,51 y 111,11 mg quercetina/g de planta. En el mismo estudio, el resultado de fenoles totales fue mayor a $100 \mathrm{mg}$ ácido gálico/g extracto seco casi cuatro veces más a nuestro extracto hidroalcohólico de $75 \%$ con $24,89 \mathrm{mg}$ ácido gálico/g planta. Otros estudios de flavonoides realizados a extractos metanólicos de hojas de Buddleja saligna ${ }^{9}$ determinaron 1,61 mg de quercetina/g de planta seca comparado con el valor obtenido de los extractos estudiados entre 118,52 a $369,24 \mathrm{mg}$ de quercetina/g planta. Un estudio realizado solo a extractos etanólicos de la Buddleja inkana ${ }^{18}$ de dos diferentes lugares de Ecuador reportó para fenoles totales valores entre 27,47- 47,40 mg de ácido gálico/g de extracto, comparados a los obtenidos entre 82,29 - 172,58 mg de ácido gálico/g de extracto.

\section{CONCLUSIONES}

Los extractos hidroalcohólicos al $75 \%$ (v/v) de etanol de las hojas de Oreocallis grandiflora y de la Buddleja inkana, O 75 y B 75, respectivamente; presentaron la mayor actividad antioxidante con los tres ensayos: FRAP, DPPH y $\mathrm{ABTS}^{-+}$. Para el extracto $\mathrm{O} 75$ se obtuvo actividad antioxidante en FRAP de 1317,53 mg Trolox/g planta, en DPPH un valor de $\mathrm{IC}_{50}$ igual a 25,26 y en $\mathrm{ABTS}^{-+}$un valor de IC50 igual a $4,69 \mu \mathrm{g} / \mathrm{mL}$ y para el extracto B $75 \mathrm{se}$ obtuvo actividad antioxidante en FRAP de 1997,78 mg Trolox/g de planta, en DPPH un valor de $\mathrm{IC}_{50}$ igual a $62,39 \mu \mathrm{g} / \mathrm{mL}$ y en ABTS ${ }^{+}$un valor de IC50 igual a $6,54 \mu \mathrm{g} / \mathrm{mL}$.

El extracto hidroalcohólico $\mathrm{O} 75$ presentó la mayor actividad antioxidante. El comportamiento antioxidante guardó relación con el contenido de compuestos fenólicos lo cual se evidenció a través de la cuantificación de metabolitos secundarios: flavonoides y fenoles totales. 


\section{AGRADECIMIENTO}

Los autores agradecen el apoyo al CONCYTEC-FONDECYT por el financiamiento del proyecto 155-2017- FONDECYT, así como la colaboración del Dr. Mario Simirgiotis y la Ing. Milena Ríos, del Laboratorio de Productos Naturales de la Facultad de Farmacia de la Universidad Austral de Chile y del Dr. Carlos Areche, del Laboratorio de Química de Compuesto Naturales Extremos de la Facultad de Ciencias de la Universidad de Chile.

\section{REFERENCIAS BIBLIOGRÁFICAS}

1. García J, Laos D, Vega N, Bendezú M, Yarasca P, Guillermo J, et al. Actividad antioxidante y antimicrobiana del extracto etanólico de las partes aéreas de Solanum radicans L.F. "huallpachaqui”. Rev Soc Quim Perú. 2020; 86(1): 5-12.

2. Castañeda B, Ramos E, Ibañez L. Evaluación de la capacidad antioxidante de siete plantas medicinales peruanas. Horiz Med (Lima). 2008; 8(1): 56-72.

3. Alam M, Juraimi A, Rafii M, Abdul-Hamid A, Aslani F, Hasan M, et al. Evaluation of antioxidant compounds, antioxidant activities, and mineral composition of 13 collected purslane (Portulaca oleracea L.) Accessions. Biomed Res Int. 2014;2014:296063. doi: $10.1155 / 2014 / 296063$.

4. Lee JH, Lee SJ, Park S, Kim HK, Jeong WY, Choi JY, et al. Characterisation of flavonoids in Orostachys japonicus A. Berger using HPLC-MS/MS: Contribution to the overall antioxidant effect. Food Chem. 2011; 124(4): 1627-1633.

5. Apak R, Güçlü K, Demirata B, Özyürek M, Çelik S, Bektaşoğlu B. Comparative Evaluation of Various Total Antioxidan Capacity Assay to Phenolic Compounds with the CUPRAC Assay. Molecules. 2007; 12: 1496-1547.

6. Ordóñez E, López A, Reátegui D. Infusiones de plantas medicinales: Actividad antioxidante y fenoles totales. Agroind Sci. 2020; 10(3): 259-266.

7. Lock O. Investigación fitoquímica: Métodos en el estudio de productos naturales. Lima: Fondo Editorial de la Pontificia Universidad Católica del Perú; 1988.

8. Morales G, Paredes A. Antioxidant activities of Lampaya medicinalis extracts and their main chemical constituents. BMC Complement Altern Med. 2014 Jul 21;14:259. doi: 10.1186/1472-6882-14-259.

9. Adedapo AA, Jimoh FO, Koduru S, Masika PJ, Afolayan AJ. Assessment of the medicinal potentials of the methanol extracts of the leaves and stems of Buddleja saligna. BMC Complement Altern Med. 2009; 9:21. doi: 10.1186/1472-6882-9-21.

10. Twilley D, Moodley D, Rolfes H, Moodley I, McGaw LJ, Madikizela B, et al. Ethanolic extracts of South African plants, Buddleja saligna Willd and Helichrysum odoratissimum (L.) sweet, as multifunctional ingredients in sunscreen formulations. S Afr J Bot. 2021;137:171-182.

11. Backhouse N, Rosales L, Apablaza C, Goïty L, Erazo S, Negrete R, et al. Analgesic, anti-inflammatory and antioxidant properties of Buddleja globosa, Buddlejaceae. J Ethnopharmacol. 2008;116(2):263-269. 
12. Pendota S, Ndhala A, Aremu A, Aderogba M, Van Staden J. Anti - inflammatory, antioxidant and in silico studies of Buddleja salviifolia (L). Lam leaf constituents. S Afr J Bot. 2014; 93: 79-85.

13. Alejandro M, Jaramillo X, Ojeda S, Malagon O, Ramirez J. Actividad antioxidante y antihiperglucemiante de la especie medicinal Oreocallis grandiflora (Lam.) R. Br., al sur de Ecuador. Bol Latinoam Caribe Plantas Med Aromát. 2013; 12 (1): 59-68.

14. Sánchez M, Anicama N. Contenido de polifenoles totales y actividad antioxidante in vitro del extracto etanólico de hojas y flores de Chuiraga spinosa Less "huamanpinta" [Tesis]. Ica: Universidad San Luis Gonzaga de Ica; 2015.

15. Casado R, Landa A, Calvo J, García-Mina JM, Marston A, Hostettmann K, et al. Antiinflammatory, antioxidant and antifungal activity of Chuquiraga spinosa. Pharm Biol. 2011; 49(6):620-626.

16. Dobravalskyte D, Venskutonis PR, Talou T. Antioxidant properties and essential oil composition of Calamintha grandiflora L. Food Chem. 2012;135(3):1539-46.

17. Herrera O, Tinco J, Arroyo J, Franco C, Chumpitaz V, Castro W, et al. Antioxidant activity and cytotoxic profile of Chuquiraga spinosa Lessing on human tumor cell lines: Apromissory plant from Peruvian flora. Asian Pac J Trop Dis. 2017; 7 (5): 304-308.

18. Llanga Guamán. Determinación de la actividad antioxidante de los extractos de Quishuar (buddleja incana), Aliso (Alnus acuminata) y Romerillo (Hypericum laricifolium) localizados en 3 zonas geográficas diferentes. [Tesis de grado]. Riobamaba, Ecuador: Escuela Superior Politécnica de Chimborazo; 2014. [Recuperado el 10 de marzo del 2021]. Disponible en: http://dspace.espoch.edu.ec/bitstream/123456789/3427/1/5 6T00446.pdf 